This document is the accepted manuscript version of the following article:

Maguire, C. M., Sillence, K., Roesslein, M., Hanne11, C., Suarez, G., Sauvain, J. J., Capracotta, S., Conta1, S., Cambier, S., E1 Yamani, N., Dusinska, M., Dybowska, A., Vennemann, A., Cooke, L., Haase, A., Luch, A., Wiemann, M., Gutleb, A., Korenstein, R., ... Prina-Me11o, A. (2017). Benchmark of nanoparticle tracking analysis on measuring nanoparticle sizing and concentration. Journal of Micro and Nano-Manufacturing, $5(4), 041002$ (10 pp.). https://doi .org/10.1115/1.4037124

\title{
Benchmark of Nanoparticle Tracking Analysis on measuring nanoparticle sizing and concentration
}

\author{
Ciarán M. Maguire \\ Nanomedicine and Molecular Imaging Group, AMBER Centre and CRANN Institute, \\ Trinity College Dublin \\ Department of Clinical Medicine, Trinity Translational Medicine Institute, Trinity Centre \\ for Health Sciences, St. James's Hospital, James Street, Dublin8, Ireland \\ cmmaguir@tcd.ie
}

\section{Katherine Sillence}

Malvern Instruments Ltd

Minton Park, London Road, Amesbury, Wiltshire, SP4 7RT, UK

ksillence@hotmail.co.uk

\section{Matthias Roesslein}

EMPA

Swiss Federal Laboratories for Materials Research \& Testing, Laboratory for Particles -

Biology Interactions, Lerchenfeldstrasse 5, CH-9014 St Gallen, Switzerland

matthias.roesslein@empa.ch

\author{
Claire Hannell \\ Malvern Instruments Ltd \\ Minton Park, London Road, Amesbury, Wiltshire, SP4 7RT, UK \\ clairehannell@gmail.com
}

\author{
Guillaume Suarez \\ Institute for Work and Health (IST) \\ Switzerland, Rte de la Corniche 2, 1066 Epalinges, Lausanne1011, Switzerland. \\ guillaume.suarez@chuv.ch \\ Jean-Jacques Sauvain \\ Institute for Work and Health (IST) \\ Switzerland, Rte de la Corniche 2, 1066 Epalinges, Lausanne1011, Switzerland. \\ jean-jacques.sauvain@hospvd.ch
}

\section{Sonja Capracotta}

Malvern Instruments Ltd

1415 Washington Heights, Rm 6611, Ann Arbor, MI 48109, United States.

sonja.capracotta@malvern.com 


\section{Servane Contal}

Luxembourg Institute of Science and Technology (LIST)

Environmental Research and Innovation (ERIN) Department, 41, rue du Brill, L-4422

Belvaux, Grand-duchy of Luxembourg

servane.contal@list.lu

\section{Sebastien Cambier}

Luxembourg Institute of Science and Technology (LIST)

Environmental Research and Innovation (ERIN) Department, 41, rue du Brill, L-4422

Belvaux, Grand-duchy of Luxembourg

sebastien.cambier@list.lu

\section{Naouale El Yamani}

Health Effect Laboratory

MILK, Norwegian Institute for Air Research, Instituttveien 18 P.O. Box 100, Kjeller, NO-

2027, Norway.

elyamani.naouale@nilu.no

\section{Maria Dusinska}

Health Effect Laboratory

MILK, Norwegian Institute for Air Research, Instituttveien 18 P.O. Box 100, Kjeller, NO2027, Norway.

maria.dusinska@nilu.no

\section{Agnieszka Dybowska}

Department of Earth Sciences

Natural History Museum, Cromwell Road, London, SW7 5BD, UK

a.dybowska@nhm.ac.uk

\section{Antje Vennemann}

IBE R\&D Institute for Lung Health gGmbH

Mendelstrasse 11, 48149 Münster, Germany

vennemann@ibe-ms.de

\section{Laura Cooke}

Centre for Bio-Nano Interactions (CBNI)

University College Dublin, Conway Institute, Belfield, Dublin 4, Ireland

laura000cooke@gmail.com

\section{Andrea Haase}

German Federal Institute for Risk Assessment (BfR)

Department of chemicals and product safety, Max-Dohrn-Strasse 8-10, 10589 Berlin, Germany

andrea.haase@bfr.bund.de 


\section{Andreas Luch}

German Federal Institute for Risk Assessment (BfR)

Department of chemicals and product safety, Max-Dohrn-Strasse 8-10, 10589 Berlin, Germany

andreas.luch@bfr.bund.de

\section{Martin Wiemann}

IBE R\&D Institute for Lung Health gGmbH

Mendelstrasse 11, 48149 Münster, Germany

martin.wiemann@ibe-ms.de

\section{Arno Gutleb}

Luxembourg Institute of Science and Technology (LIST)

Environmental Research and Innovation (ERIN) Department, 41, rue du Brill, L-4422

Belvaux, Grand-duchy of Luxembourg

arno.gutleb@list.lu

\section{Rafi Korenstein}

Dept of Physiology \& Pharmacology, Sackler School of Medicine, Tel Aviv, Tel 69978, Israel

korens@post.tau.ac.il

\section{Michael Riediker}

Institute of Occupational Medicine Singapore Pte. Ltd., 30 Raffles Place, \#17-08 Chevron House, Singapore, 048622

Singapore

michael.riediker@iom-world.sg

\section{Peter Wick}

Swiss Federal Laboratories for Materials Research \& Testing, Laboratory for Particles Biology Interactions, Lerchenfeldstrasse 5, CH-9014 St Gallen, Switzerland peter.wick@empa.ch

\section{Patrick Hole}

Malvern Instruments Ltd

Minton Park, London Road, Amesbury, Wiltshire, SP4 7RT, UK

patrick.hole@malvern.com

\section{Adriele Prina-Mello ${ }^{1}$}

\footnotetext{
${ }^{1}$ Email: prinamea@tcd.ie, Telephone: +353 (1) 8963259

Department of Clinical Medicine, Trinity Translational Medicine Institute, Trinity Centre for Health Sciences, St. James's Hospital, James Street, Dublin8, Ireland
} 
Nanomedicine and Molecular Imaging Group, AMBER Centre and CRANN Institute, Trinity College Dublin

Department of Clinical Medicine, Trinity Translational Medicine Institute, Trinity Centre for Health Sciences, St. James's Hospital, James Street, Dublin8, Ireland

prinamea@tcd.ie 


\begin{abstract}
One of the greatest challenges in the manufacturing and development of nanotechnologies is the requirement for robust, reliable and accurate characterization data. Presented here are the results of an interlaboratory comparison brought about through multiple rounds of engagement with NanoSight Malvern and 10 pan-European research facilities. Following refinement of the Nanoparticle Tracking Analysis technique, the size and concentration characterization of nanoparticles in liquid suspension was proven to be robust and reproducible for multiple sample types in mono-modal, binary or multi-modal mixtures. The limits of measurement were shown to exceed the $30 \mathrm{~nm}$ to $600 \mathrm{~nm}$ range (with all system models), with percentage coefficients of variation being calculated as sub $5 \%$ for monodisperse samples. Particle size distributions were also improved through the incorporation of the Finite Track Length Adjustment algorithm, which most noticeably acts to improve the resolution of multimodal sample mixtures. The addition of a software correction to account for variations between instruments also dramatically increased the accuracy and reproducibility of concentration measurements. When combined, the advances brought about during the ILCS allow for the simultaneous determination of accurate and precise nanoparticle sizing and concentration data in one measurement.
\end{abstract}

\title{
INTRODUCTION
}

Accuracy, reliability, reproducibility, and robustness are fundamental parameters when it comes to the characterisation of nanomaterials. It is essential to be able to obtain sound, statistical data for a sample that is not overly influenced by outliers, while giving true and accurate reflection of the entire sample population. In terms of nanoparticle size and concentration determination, results obtained must reflect the entire sample, and should not be influenced by outliers or aggregates, while also not excluding these results. Deficits in these areas can have implications in nanomaterial manufacturing, quality control, and regulatory approval, where the physical-chemical properties of a material must be known. Where a synthetic or development process 
requires the use of strict tolerances, the technique used must be validated to accurately characterise the sample and obtain results within these values. The determination of confidence intervals and calculations of measurement error is therefore crucial.

The importance of reproducible scientific research [1] and the need for "reproducible, transparent and independent verification of the scientific method" to improve the quality control in research $[2,3]$ has been identified by publishers and the scientific community alike. More data from a recent survey by Nature Publishing Group has shown that of 1,576 researchers surveyed, $34 \%$ have no guidelines in place to improve reproducibility [4].

Within the field of particle characterisation, a series of interlaboratory comparisons (ILCS) were conducted in order to establish an understanding for the level of reproducibility end-users could achieve with their Nanoparticle Tracking Analysis (NTA) systems. The use of an ILC allows for monitoring the ability of a laboratory to deliver accurate testing results, or to identify if a particular analytical method is suitable for its intended purpose. Using agreed and standardised procedures, and calibration standards, the validity or accuracy of a technique can also be determined [5].

The technique utilises the properties of nanoparticle light scattering and Brownian motion to determine the size distribution of a sample in liquid suspension [5], characterising the sample on a particle-by-particle basis. As such the size-distributions are not weighted towards larger particle sizes, a feature commonly associated with NTA comparative technique Dynamic Light Scattering (DLS). A laser beam, typically 405, 488, 
532 or $635 \mathrm{~nm}$ in wavelength, is passed through a sample chamber where the particles in suspension in the path of the beam scatter light which is visualised using video camera mounted to a $\times 20$ magnification microscope. The Brownian motion of the particles is recorded in a series of videos, with the software using a modified Stokes-Einstein equation to calculate the hydrodynamic diameters of each individual nanoparticle [5]. The counting of individual particles also enables the concentration of the sample in nanoparticles per millilitre (NPs/mL), to be simultaneously determined as the volume of the field of view is known. These features have also allowed the NTA technique to be developed as ASTM [6] and ISO standards [7] for the measurement of particle size distributions.

NTA is rapidly becoming a fundamental technique for the characterisation of nanoparticles from a multitude of sources, both engineered and naturally occurring nanoparticles alike. An analysis of the database Scopus shows that since 2007, there have been 160 documents that site the NTA technique, covering areas such as chemistry, medicine biochemistry and molecular genetics (Figure 1).

Figure 1

Similarly, initial studies on the concentration determination of a nanoparticle suspension yielded results that were seen to differ greatly amongst laboratories, and was identified as opportunity for the improvement of the NTA system (Figure 2).

Figure 2 
Currently in the nanosafety field there is a high need to develop characterisation techniques that comply with directives, guidelines and regulations as requested by regulatory agencies. This work supports the NTA technology to become an accepted standard benchtop characterisation technique for any product which will have to be certified to contain nano-objects, as required by EC directives [8-10].

Present here are the results of two consecutive rounds of ILCs where we aim to refine and validate the NTA technique, thought the use of standardised procedures distributed by Malvern Instruments Ltd. (Malvern) and improved software features. Similarly, we aim to calculate the coefficients of variation and measurement errors inherent in NTA systems covering a wide array of potential setups.

\section{Experimental Section}

The improvement of the size and concentration determination of nanoparticles in suspension by NTA was carried out in two distinct ILCs (ILC 2 and ILC 3) which carry on from an original comparison study [5] (ILC 1). 10 laboratories participated in this study with the NTA systems utilised in this study being listed in Table 1 . Due to some laboratories being unable to participate in all rounds, participant numbers in some figures are not equal. Similarly, some laboratories tested more than one NanoSight system. Participant numbers are also randomised, such that lab 1 in one figure may not be lab 1 in the next.

Briefly, ILC 1 determined the optimal video recording duration (minimum of five x 60 second videos) and allowed users to identify the most optimal nanoparticle 
dilutions (between 20-100 NPs per field of view) to enable the most accurate measurements. Samples that are too dilute result in fewer particles tracked, with too concentrated samples resulting in incomplete particle tracking due to particle tracks merging together. Other improvements included reducing the number of user changeable settings to reduce the number of parameter variations between users from $3.8 \times 10^{23}$ to $1.6 \times 10^{3}[5]$.

ILC 2 introduced the Finite Track Length Adjustment (FTLA) algorithm which uses data processing methods and statistics to improved peak isolation [11]. Here, FTLA algorithm accounts for the tracking of a particle over a finite number of frames leading to a statistical error in the average particle diameter [11]. Various beta versions of new NTA software were tested by partners in this study to validate the results obtained, leading to the introduction of the versions currently available.

ILC 3 involved the individual calibration of each NanoSight system and applying a calibration factor obtained directly from Malvern, henceforth called "software correction". This will account for variations in laser and camera setup for each NanoSight system, thereby allowing each user to obtain the theoretical concentration values calculated by Malvern. This software correction is machine specific, and proprietary, as the concentration upgrade is a commercial product available for purchase. Again, various beta-version of the NTA software were tested and validated, with results being collated and analysed by Malvern. 
The study design also encompassed many aspects of the principles and guidelines suggested by the National Institute of Health $(\mathrm{NIH})$ to deal with the reproducibility of research, including the use of rigorous statistical analysis, standards, replicates and randomisation in order to develop best practice guidelines [12].

$30 \mathrm{~nm}$ gold (BBI Solutions, UK), and 100, 200, 300, 400 and $600 \mathrm{~nm}$ polystyrene latex spheres (Thermoscientific, UK) for use in size distribution experiments (ILC 2), and 60, 100, 200 and $300 \mathrm{~nm}$ polystyrene latex spheres (Thermoscientific, UK) for concentration experiments (ILC 3), with characteristics listed below in Table 2, were shipped to laboratories with temperature sensors to indicate if NPs were exposed to temperatures below $4{ }^{\circ} \mathrm{C}$ or above $29^{\circ} \mathrm{C}$. Samples were analysed first by Malvern, followed by the shipment of aliquots from the stock solution to participants. Samples for concentration measurements were diluted by Malvern before shipping, and instructions on dilution factors were provided for sizing measurements based on prior analysis by Malvern. Laboratories were supplied with a range of disposable sample tubes, $0.02 \mu \mathrm{m}$ syringe filters and HPLC grade water (Rathburn Chemicals Ltd, UK; product no: RH1020). Software updates and recalibration protocols were issued to participants as required. All samples were recorded for 60 seconds with 6 replicates, to improve statistical analysis. Camera levels and detection threshold values were set using values deemed optimal by each individual user. This ensures that only distinct particles with minimal defraction rings were visible, and tracked correctly based on their individual system setup. Between 10-100 distinct particles should be marked with red crosses by the software per frame of each video. Red crosses that are not due to distinct particles should be less 
than 10. Different system settings are required depending on the size, refractive index, and ultimately the light scattering properties of the materials tested. Fresh sample was injected into the sample chamber after each recording to introduce new particles to be tracked and analysed. This was carried out manually using $1 \mathrm{~mL}$ syringes on LM10 and LM20 systems, and automatically using the fluidics controls on the NS500 systems. Viscosity tables were provided for the $10 \%$ glycerol (>99 \%, molecular biology grade, Sigma Aldrich, UK) solution which was required by the software as part of the StokesEinstein equation to calculate NP diameter. For the mixed population samples, a ratio of 18:3:2 (100 nm: $200 \mathrm{~nm}: 300 \mathrm{~nm})$ and 6:3:3:2 (100 nm: $200 \mathrm{~nm}: 300 \mathrm{~nm}: 400 \mathrm{~nm}$ ) was used. For the trimodal samples this equated to an approximate concentration of $4.24 \mathrm{x}$ $10^{8}: 7.07 \times 10^{7}: 4.72 \times 10^{7}$ particles, with the quadrimodal sample comprising $8.96 \times 10^{7}$ : $4.48 \times 10^{7}: 4.48 \times 10^{7}: 2.98 \times 10^{7}$ particles, as calculated by the manufacturer's specifications. A single user from each laboratory conducted the analysis of all samples to assess the user variation. Software corrections for use in ILC 3 were applied based on a calibration set provided by Malvern and was unique to each system.

TABLE 2

\section{Results and Discussion}

Following extensive rounds of ILCs, Malvern Instruments Ltd, in conjunction with 10 pan-European partners, have made a number of improvements in the size characterisation of nanoparticles [5]. Most notably this comes in the form of improved limits of detections (LOD) for samples measured with all available platforms and the 
ability to analyse more complex multimodal mixtures with higher resolution through improved SOPs covering data acquisition and analysis and processing techniques.

Through technical advances in the proprietary software, the resolution accessible using all available instrument platforms and system combinations (including the least sensitive systems) has been demonstrated to cover the range of $30 \mathrm{~nm}$ to 600 $\mathrm{nm}$. Within this range, nanoparticle size distribution data can be accurately and reproducibly acquired as indicated in figures $3 a$ and $3 b$ for $30 \mathrm{~nm}$ gold and $600 \mathrm{~nm}$ polystyrene latex spheres (PSL), respectively. Reflected here are the median and median absolute deviations (MAD) values which demonstrate the variations in size obtained for the particles across participants. Coefficients of variation of $3.95 \%$ for gold NPs and $4.01 \%$ for $600 \mathrm{~nm}$ PSL NPs were obtained. These results also correlate very well with the TEM sizes obtained from the manufacturer (Table 2). It should be noted that the upper and lower operating limits presented are laser and camera system dependent. High sensitivity camera and high energy laser setups will result in large particles, such as the $600 \mathrm{~nm}$ PSL NPs appearing very bright on screen, making focusing and NP tracking more difficult. This ultimately will increase user variation. Similarly, low sensitivity cameras and lower powered lasers will reduce the ability to detect smaller particles. Thus, presented here, are results and variations that could be typically expected for any NTA system used.

\section{FIGURE 3}

The ability of NTA to track and analyse nanoparticles on the lower LOD was further interrogated through alteration of the viscosity of the solvent, figure 4. Based on the Stoke-Einstein equation, once the viscosity of the solution $(\eta)$ is known and particle 
diffusion coefficient $\left(D_{t}\right)$ is measured, the particle diameter can be calculated [5]. As such, the addition of $10 \% \mathrm{w} / \mathrm{v}$ glycerol to a solution of $30 \mathrm{~nm}$ gold particles increased the viscosity of the sample, slowing the Brownian motion of the particles and thus allowing for enhanced nanoparticle tracking (figure 4A and 4B). This is particularly evident for the samples measured in glycerol where the median absolute deviation (MAD) showed a reduction from $3.2 \mathrm{~nm}$ to $2.5 \mathrm{~nm}$.

\section{FIGURE 4}

Improved analysis algorithms have allowed for NTA to be used in the characterisation of complex multimodal systems, an improvement from the bimodal detection present in ILC 1[5]. This multimodal capability opens up a wider range of real world applications, with particular emphasis on industry. In line with EU nanoparticle regulations, all nanoparticles produced either directly or indirectly must be fully characterised [13]. However, from a quality control basis, the ease of use of the NTA system allows for characterisation of engineered nanoparticles throughout various stages of production. The ability of the NTA software to resolve multimodal mixes at different ratios of nanoparticles as depicted in figure $5 a-d$, has the added benefit in detecting nanoparticle synthesis difficulties at an early stage and therefore provides a reliable tool for quality control and assurance. What it can be clearly seen here is the ability of each NTA system to resolve the tri- and quadri-modal samples used as a reflection of polydisperse samples. The dashed line in figures $5 a$ and $c$ represent the median value obtained for each component in the mixture, with the shaded areas in figures $5 \mathrm{~b}$ and $\mathrm{d}$ illustrating the interlaboratory variation. 


\section{FIGURE 5}

The ability to improve the analysis of multimodal and polydisperse samples compared to the initial ILC [5], is primarily due to the implementation of Finite Track Length Adjustment (FTLA). This data processing algorithm can be applied to compensate for the randomness of Brownian motion [11]. FTLA allows for improved peak isolation allowing for the improved sizing of multiple components in a multimodal sample, as shown in figure 6. As can be seen, when FTLA is applied (solid line), the individual peaks for the components of the sample can be resolved in greater detail, compared to the broad peaks prior to applying FTLA.

\section{FIGURE 6}

Stringent compliance with SOPs developed throughout the course of this work has allowed for a reduction in the \% coefficient of variance (\% CV) for samples covering a wide portion of the Nanosight's LOD, as demonstrated in figure 7A. Dispersion of 30 nm gold particles in $10 \%$ glycerol resulted in a reduction in \% CV from 3.945, in water, to 3.545 , with a corresponding median absolute deviation (MAD) \% CV reduction from 0.904 to 0.897 . Similarly, \% CV [MAD \% CV] of 1.69 [0.54], 0.59 [0.548] and 4.01 [2.15] were obtained for 100, 200 and $600 \mathrm{~nm}$ polystyrene particles, which shows dramatic improvement on the original ILC [5].

The nature of this interlaboratory study was such that it was also possible to account for instrumentation specifications variation as shown in figure 7A and B where inter-user effects are incorporated, resulting in a larger CV, as also reported in the previous study [5]. Analysis of multimodal samples also has implication for the 
reproducibility of results and the $\% \mathrm{CV}$. Figure $7 \mathrm{~B}$ below demonstrates the spread of the $\% \mathrm{CV}$ for each laboratory following the analysis of two mixed nanoparticle samples: (i) a 100, 200 and $300 \mathrm{~nm}$ mix of polystyrene nanoparticles, and (ii) a 100, 200, 300 and 400 $\mathrm{nm}$ mixture. The $100-300 \mathrm{~nm}$ samples resulted in a lower $\% \mathrm{CV}$ for all populations, with an increased $\%$ CV being observed for the $100-400 \mathrm{~nm}$ mixture. Interestingly, the larger NPs had an overall lower \% CV. For the 100-300 nm mixture, a mean \% CV of 4.94, 2.88 and 2.43 were obtained for the 100, 200 and $300 \mathrm{~nm}$ components, respectively. Similarly, for the $100-400 \mathrm{~nm}$ mixture, the mean \% CV observed was $6.82,3.88,2.60$ and 3.09 for the $100,200,300$ and $400 \mathrm{~nm}$ polystyrene NPs, respectively.

\section{FIGURE 7}

The accuracy and linearity of the NTA concentration measurements improved greatly upon application of the software correction. Standard $100 \mathrm{~nm}$ PSL samples gave a linear response over the range from $8.6 \times 10^{6}$ to $5.7 \times 10^{9}$ particles per $\mathrm{mL}$ as shown in Figure 8. This covers the optimal NTA concentration range, allowing accurate, reproducible concentration results at the extremes of concentration for accurate NTA particle sizing. Data (not shown) from one ILC sub-round replicated these improvements over a narrower concentration range for both 100 and $300 \mathrm{~nm}$ PSL, showing the reduction in the variability of concentration measurements with user selected settings.

\section{FIGURE 8}

Fundamentally, in this study, improved protocols and software provided an increase in accuracy and precision for the concentration measurements and reduced sensitivity to user selected settings. Following the software correction, a significant 
reduction in the variation of the nanoparticle concentration measured was observed, as reported in Figure 9. Depicted here are the variations in concentration measurements for $60,100,200$ and $300 \mathrm{~nm}$ samples, before and after the machine specific software correction. The solid line in the figure reflects the theoretically calculated particle concentration, with the grey shading represents $95 \%$ confidence interval.

\section{FIGURE 9}

The impact of the software correction development was also assessed for its implications on the ability of any NanoSight system to robustly measure both nanoparticle concentration and sizing simultaneously. As is clearly illustrated following software correction, results from all labs are centred on the respective calculated concentrations. No changes in particle sizing were observed following the upgrade, as shown in Table 3. This result allows users of NTA to accurately determine both size and concentration of their particle suspension in one measurement, with the potential to speed up characterisation workflows.

\section{TABLE 3}

Through refinement of the SOPs during three rounds of ILCs, the accuracy and reproducibility of NTA has been refined, as illustrated in Figure 10. Initial assessment before ILC 1 demonstrated that there was a large percentage error in both sizing and concentration measurements, a problem that needed to be addressed (Figure 10-top panel). Subsequently, during ILC 1 and within the ILC 2 NTA software advances and stringent protocols brought about a dramatic reduction in NTA sizing error. However, a large percentage error in concentration measurement was still detected (Figure 10- 
middle panel). Finally, during ILC 3 the software advances and measurement upgrade has resulted in a reduction in the percentage error for concentration measurements, leading to significant improvements in the accuracy and reproducibility of both size and concentration measurement. All reductions in percentage errors are quoted in Table 4.

FIGURE 10

TABLE 4

Variations amongst users will always be anticipated due to the variations in MalvernNanoSight systems, laser and camera setups used. Laser sources and camera sensor types are thought to be the main source of variation. Lower wavelength lasers, such as the $405 \mathrm{~nm}$ laser, allow for sizing of smaller particle, compared to higher wavelength versions, such as $635 \mathrm{~nm}$ lasers, due to Rayleigh scattering. Higher wavelength lasers may not detect these smaller particles as the light scattering will be reduced. Similarly, the camera sensor sensitivity is also important for the detection of smaller, lower reactive index particles. The results obtained in this study best demonstrate the variability that can be expected for the various NTA systems available.

\section{Conclusions}

This work strives to address the issues of accuracy and reproducibility in nanoparticle size and concentration characterisation using NTA. Advancements brought about this study has led to the reduction in user variability in the simultaneous measurement of nanoparticle size and concentration. For example, a $100 \mathrm{~nm}$ latex sample, at a concentration of $3.4 \times 10^{8}$ particles per $\mathrm{mL}$, was measured, by different users, using different systems and settings, to a reproducibility of $2.8 \% \mathrm{CV}$ for sizing and $8.6 \% \mathrm{CV}$ for concentration measurements. 
This research is critically important, not only to add value to the nanotechnology industry which has a predicted market value of 3,000 billion US dollars by 2020 [14], but also provide a transferable platform for the scientific community. In the areas of biomedical research, the ability to accurately size and quantify nanoparticles is crucial in order to interpret the results of biological experiments. A concentration measurement error of $170 \%$, as observed prior to the software correction, could dramatically affect the results of vaccine research, for example. It is envisioned that the improved reproducibility will allow for use of NTA in a wider setting, most notably in industry and areas of quality control and assurance, and enabling compliance with EU legislation [8, $15]$. 


\section{ACKNOWLEDGMENT}

CMM, KS, MR, CH, GS, SC, SC, AD, AV, LC, AH carried out experimental measurements on behalf of the laboratories involved in this study.

SC, $A H, A L, M D, M W, A G, R K, M R, P W, P H, A P-M$, supervised and discussed the results across laboratories.

$\mathrm{CMM}, \mathrm{KS}, \mathrm{MR}, \mathrm{PH}, \mathrm{APM}$ drafted, revised and finalized the manuscript and circulated to all authors for approval.

$\mathrm{KS}, \mathrm{CH}, \mathrm{PH}$ tested and distributed samples, developed and provided protocols, collated results and provided feedback to all laboratories and users.

The co-authors would like to thank Dr. S. Mano, Restrack AS, Norway and Dr. L. HorevAzaria, Tel Aviv University, Israel.

\section{FUNDING}

All authors would like to thank the European Union for QNano (QualityNano) -A panEuropean infrastructure for quality in nanomaterials safety testing, FP7INFRASTRUCTURES-2010-1 (Grant Agreement No. 262163).

The contribution of LIST was partially funded under the NANION project (FNR/12/SR/4009651-Fonds National de la Recherche Luxembourg).

The contribution from Empa was partially funded by the Competence Centre for Materials Science and Technology (CCMX) Project Nano-Screen. 
The contribution from Trinity College was partially funded under NAMDIATREAM (grant ref \#246479) and MULTIFUN (grant ref \#262943) projects under the EC FP7 programme.

NILU work was also supported by the Research Council of Norway, the project NorNANoREG (239199/O70).

\section{NOMENCLATURE}

$\begin{array}{ll}\text { ILC } & \text { Interlaboratory Comparison } \\ \text { NTA } & \text { Nanoparticle Tracking Analysis } \\ \text { NIH } & \text { National Institute of Health } \\ \text { SOP } & \text { Standard Operating Procedure } \\ \text { LOD } & \text { Limit of detection } \\ \text { MAD } & \text { Median Absolute Deviation } \\ \text { FLTA } & \text { Finite Track Length Adjustment } \\ \text { \% CV } & \text { Percentage coefficient of variation }\end{array}$




\section{REFERENCES}

[1] Nature Editorial, 2013, "Announcement: Reducing our irreproducibility," Nature, 496(398).

[2] Nature Editorial, 2014, "Journals unite for reproducibility," Nature, 515(7).

[3] Nature Editorial, 2016, "Repetitive flaws: Strict guidelines to improve the reproducibility of experiments are a welcome move," Nature, 529(7586).

[4] Baker, M., 2016, "1,500 scientists lift the lid on reproducibility," Nature, 533(7604), pp. 452-454.

[5] Hole, P., Sillence, K., Hannell, C., Maguire, C., Roesslein, M., Suarez, G., Capracotta, S., Magdolenova, Z., Horev-Azaria, L., Dybowska, A., Cooke, L., Haase, A., Contal, S., Manø, S., Vennemann, A., Sauvain, J.-J., Staunton, K., Anguissola, S., Luch, A., Dusinska, M., Korenstein, R., Gutleb, A., Wiemann, M., Prina-Mello, A., Riediker, M., and Wick, P., 2013, "Interlaboratory comparison of size measurements on nanoparticles using nanoparticle tracking analysis (NTA)," Journal of Nanoparticle Research, 15(12), pp. 1-12.

[6] ASTM, 2012, "Standard Guide for Measurement of Particle Size Distribution of Nanomaterials in Suspension by Nanoparticle Tracking Analysis (NTA)," ASTM International, ASTM E2834-12.

[7] ISO, In Progress, "TC 24/SC4/WG7 NWIP Determination of particle size distribution - Particle Tracking Analysis,"TC 24/SC4/WG7.

[8] European Commission, 2009, "REGULATION (EC) No 1223/2009 OF THE EUROPEAN PARLIAMENT AND OF THE COUNCIL of 30 November 2009 on cosmetic products," Official Journal of the European Commission.

[9] Bleeker, E. A. J., Jong, W. H., Geertsma, R. E., Groenewold, M., Heugens, E. H. W., Koers-Jacquemijns, M., Meent, D., Popma, J. R., Rietveld, A. G., and Wijnhoven, S. W. P., 2013, "Considerations on the EU definition of a nanomaterial: science to support policy making," Regulatory Toxicology and Pharmacology, 65(1), pp. 119-125. [10] Linsinger, T. P. J., Roebben, G., Gilliland, D., Calzolai, L., Rossi, F., Gibson, N., and Klein, C., 2012, "Requirements on measurements for the implementation of the European Commission Definition of the Term "nanomaterial"," 10.2787/63490.

[11] Walker, J. G., 2012, "Improved nano-particle tracking analysis," Measurement Science and Technology, 23(6), p. 065605.

[12] National Institute of Health, 2014, "Principles and Guidelines for Reporting Preclinical Research," http://www.nih.gov/about/reporting-preclinical-research.htm. [13] Tiede, K., Hassellöv, M., Breitbarth, E., Chaudhry, Q., and Boxall, A. B. A., 2009, "Considerations for environmental fate and ecotoxicity testing to support environmental risk assessments for engineered nanoparticles," Journal of Chromatography A, 1216(3), pp. 503-509.

[14] Roco, M., 2011, "The long view of nanotechnology development: the National Nanotechnology Initiative at 10 years," Journal of Nanoparticle Research, 13(2), pp. 427 445.

[15] Organisation for Economic Co-Operation and Development, 2009, " Guidance manual for the testing of manufactured nanomaterials: OECD sponsorship programme: first revision," ENV/JM/MONO(2009)20/REV 


\section{Figure Captions List}

Fig. 1 Areas of research where Nanoparticle Tracking Analysis is being utilised. Data obtained from the Scopus database, searching nanoparticle tracking analysis, and NTA, and represents documents published from 2007 to 2016.

Fig. 2 Preliminary concentration measurements of $100 \mathrm{~nm}$ polystyrene latex (PSL) particles by laboratory prior to Interlaboratory Comparison study. Initial measurements carried out using NTA identified a large degree of variation in calculated nanoparticle concentrations (101 \% CV) and a clear need for measurement improvement.

Fig. 3 Concentration normalized particle size distribution of A: $30 \mathrm{~nm}$ gold nanospheres in water and B: $600 \mathrm{~nm}$ polystyrene latex spheres in water at concentrations suitable for NTA analysis. Solid line represents median value where dotted lines are for the upper and lower Median Absolute Deviations (+/-MAD).

Fig. 4 Robust statistical analysis of $30 \mathrm{~nm}$ gold particles in DI water $(A)$ and in $10 \%$ glycerol (B). Dashed line represents median value, solid line is mean value where dotted lines are for the upper and lower Median Absolute Deviations (+/-MAD).

Fig. 5 Multimodal size distribution graphs and comparisons of mixtures of 100, 200 and $300 \mathrm{~nm}$ polystyrene latex (PSL) NPs. A and B plots show the clear 
tri-modal quantification of PSL NPs $(100,200$ and $300 \mathrm{~nm})$ mixed at a ratio of 18:3:2 respectively. $C$ and $D$ plots show clear quadri-modal quantification of PSL NPs $(100,200,300$ and $400 \mathrm{~nm})$ at a ratio of 6:3:3:2, respectively. In figure $A$ and $C$, particle ratios are shown within each particle peak. Figure B and D report the interlaboratory variance around the chosen NPs in the mixtures.

Fig. 6 Size distribution of a multimodal sample prior to implementation of finite track length adjustment (FTLA), and following applying FTLA.

Fig. 7 Confidence intervals of variance of monodisperse and multimodal samples. A: Scatterplots of median and interquartile range of $\%$ coefficient of variance for all laboratories for $30 \mathrm{~nm}$ to $600 \mathrm{~nm}$ nanoparticles. B: Scatterplots of median and interquartile range of $\%$ coefficient of variance for mixed population samples. Dashed boxplots represent 100, 200 and $300 \mathrm{~nm}$ mixed samples where solid boxplots are for $100,200,300$, and $400 \mathrm{~nm}$ mixed samples

Fig. 8 Linearity of NTA concentration measurements for $100 \mathrm{~nm}$ PSL from 8.6x106 to 5.7x109 particles per $\mathrm{ml}$. Solid line reflected best fit line following software correction $(\mathrm{R} 2=0.991)$, with grey shading illustrating $95 \%$ confidence interval. Dashed line reflects the theoretically calculated particle concentration.

Fig. 9 Concentration measurements: robust statistical analysis of 60, 100, 200 
and $300 \mathrm{~nm}$ PSL particles before and after software correction for all participant laboratories. Solid line reflects the theoretically calculated particle concentration; grey shading represents $95 \%$ confidence interval. Error bars show the standard error of the mean (SEM) of the concentration measurement.

Fig. 10 Hitting the bullseye- Refining the reproducibility of NTA size and concentration measurements through three rounds of ILC for $100 \mathrm{~nm}$ polystyrene latex (PSL) particles. Boxes indicate the primary aim for improvement of each round of ILC. 


\section{Table Caption List}

Table 1 Nanosight systems used in this study. Participant numbers have been randomized and do not correlate with laboratory numbers in other figures.

Table 2 Nanoparticle material and size distribution data as provided by the manufacturers. Latex NPs supplied by Thermoscientific (UK), lot number: $100 \mathrm{~nm}=40549 ; 200 \mathrm{~nm}=40570 ; 300 \mathrm{~nm}=41699 ; 400 \mathrm{~nm}=38736 ;$ $600 \mathrm{~nm}=39065)$. Gold NP supplied by BBI solutions (UK), lot number: 16207. Glycerol (>99 \%) lot number: STBC1888V, product number: 95516-100ml

Table 3 Table of mean particle size \pm standard deviation and percentage coefficient of variation (\% CV) for nominally 60, 100, 200 and $300 \mathrm{~nm}$ PSL particles. No change in nanoparticle sizes were observed following software correction.

Table 4 Percentage error (\% error) and percentage CV (\% CV) for nanoparticle size and concentration for all interlaboratory comparisons. 


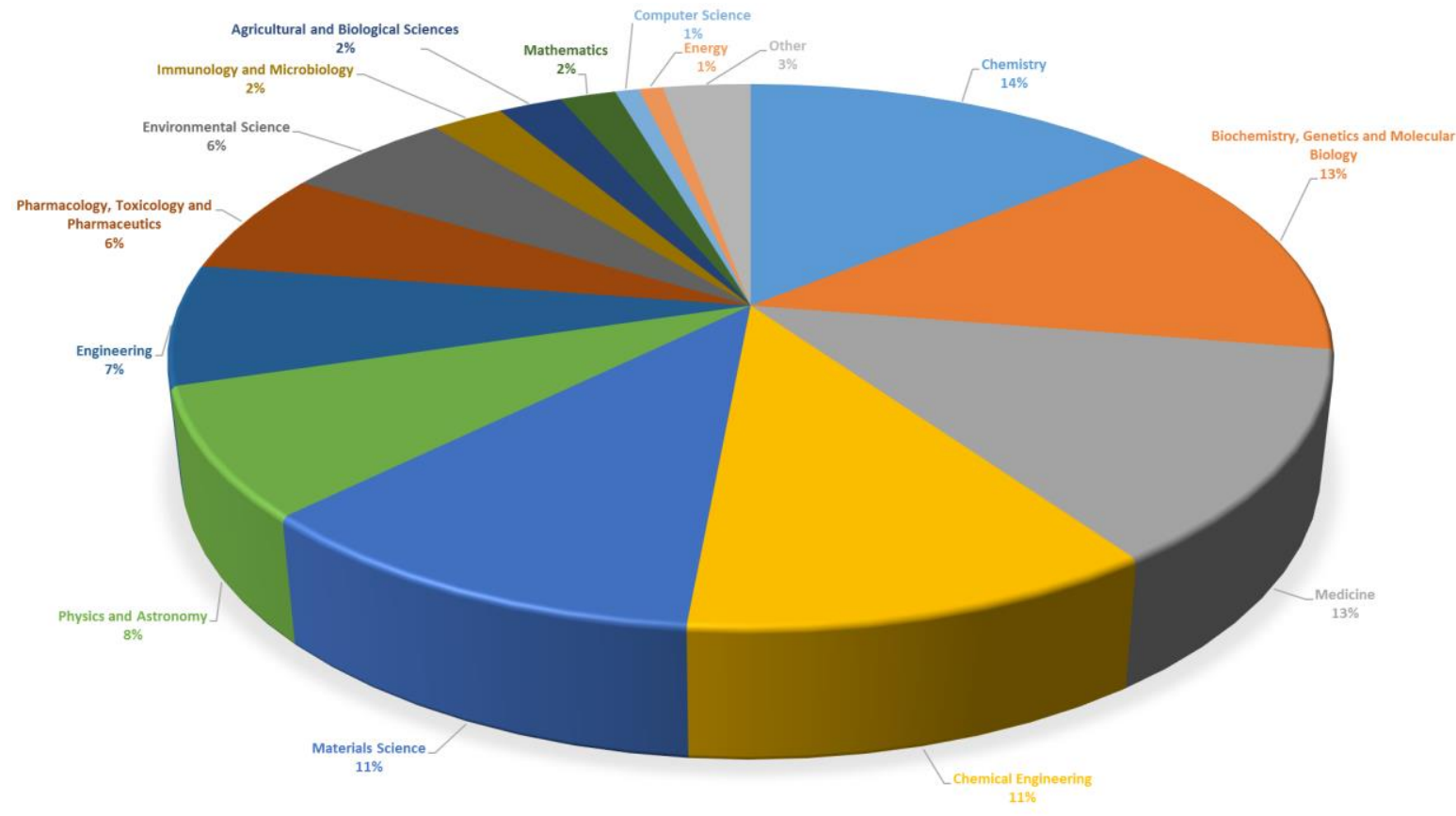

Figure1 


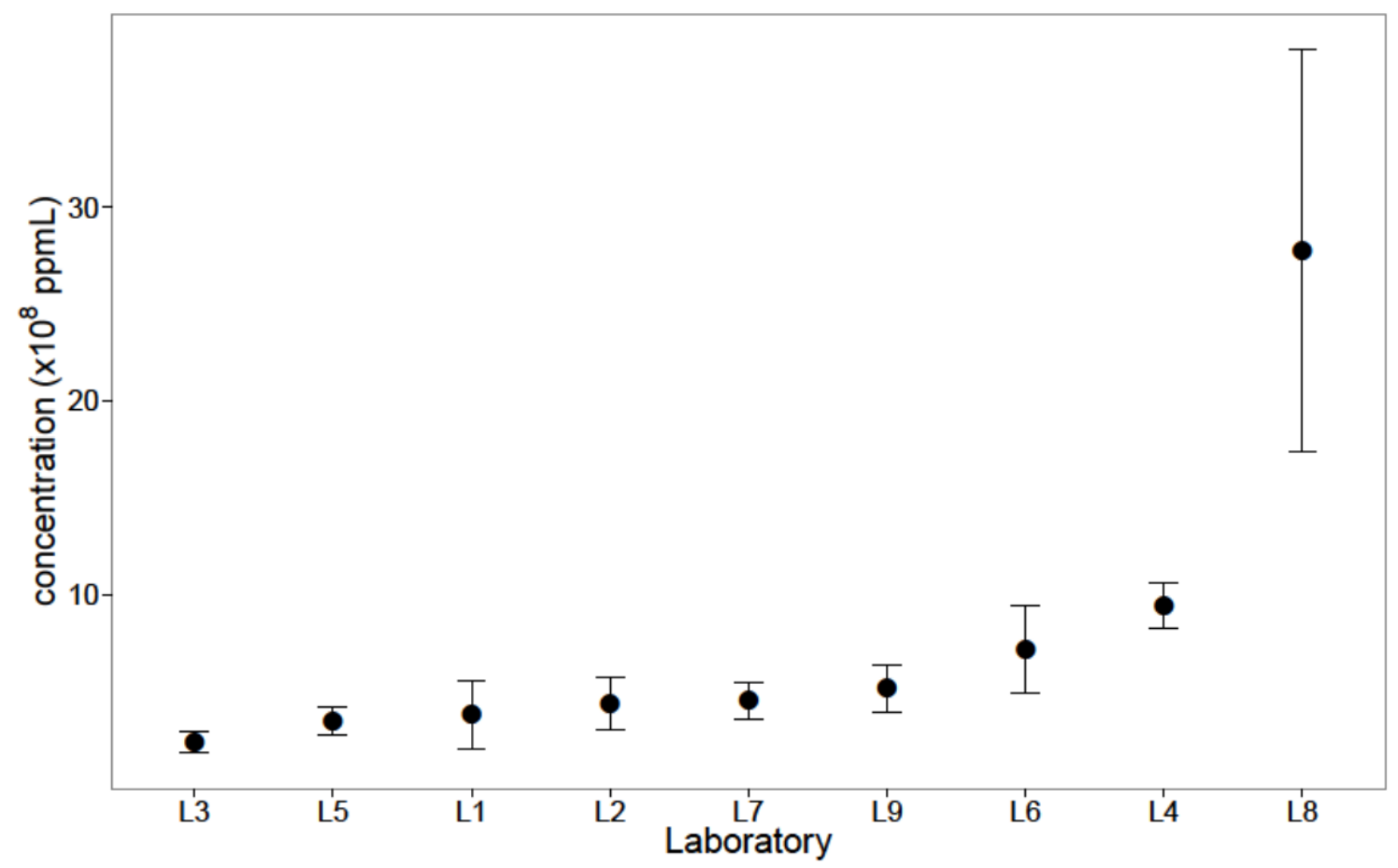

Figure 2 
A

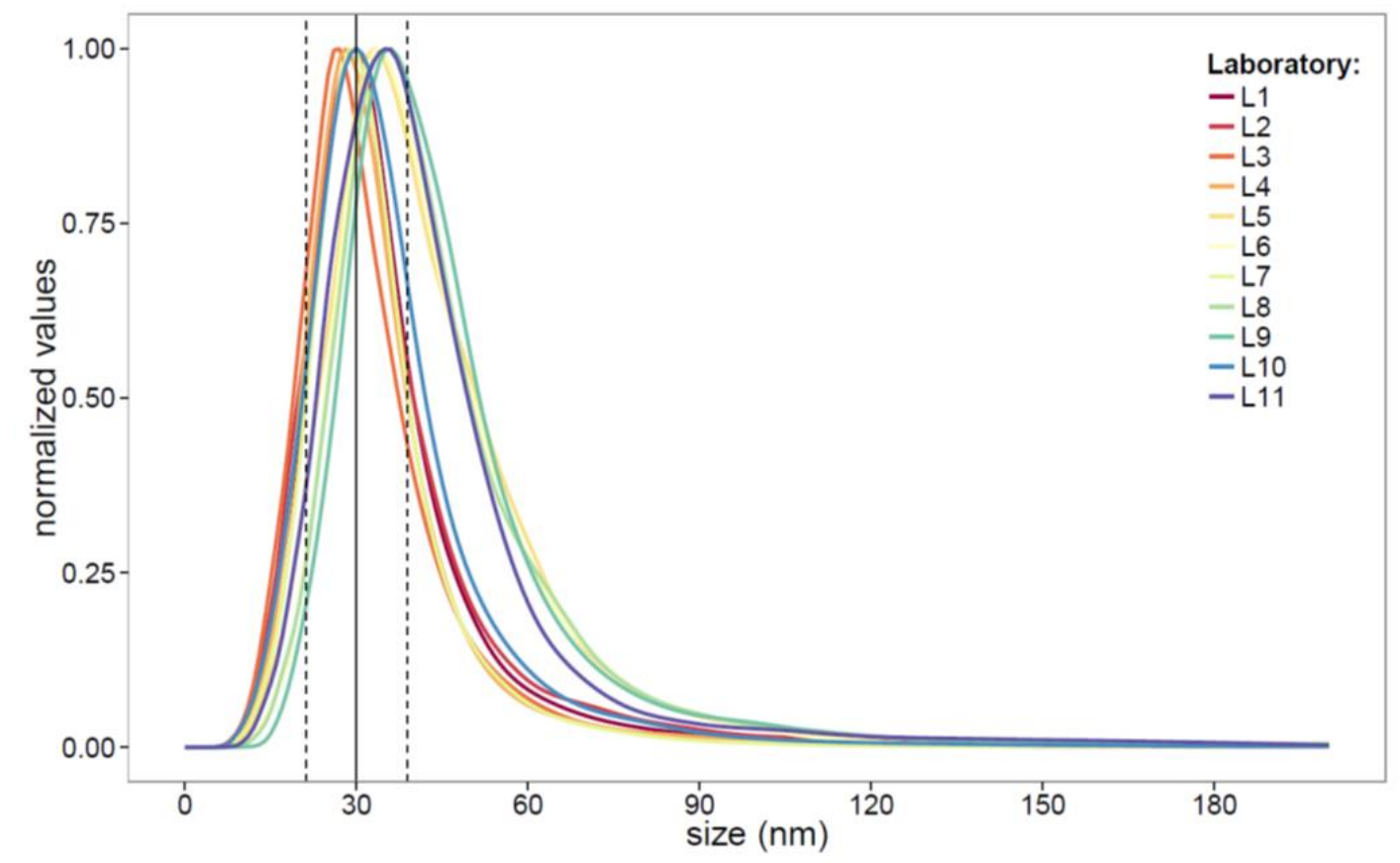

B

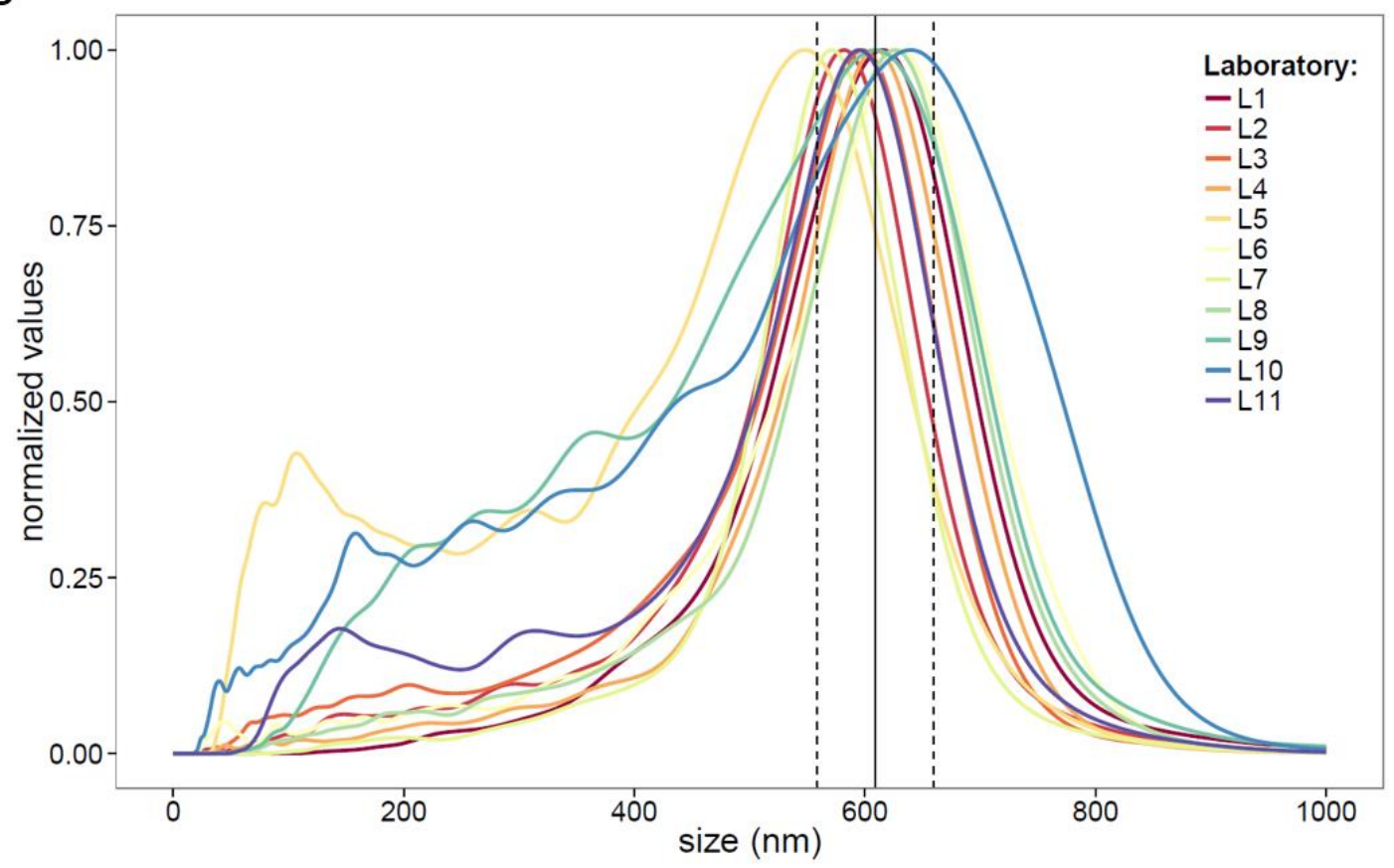

Figure 3 
A
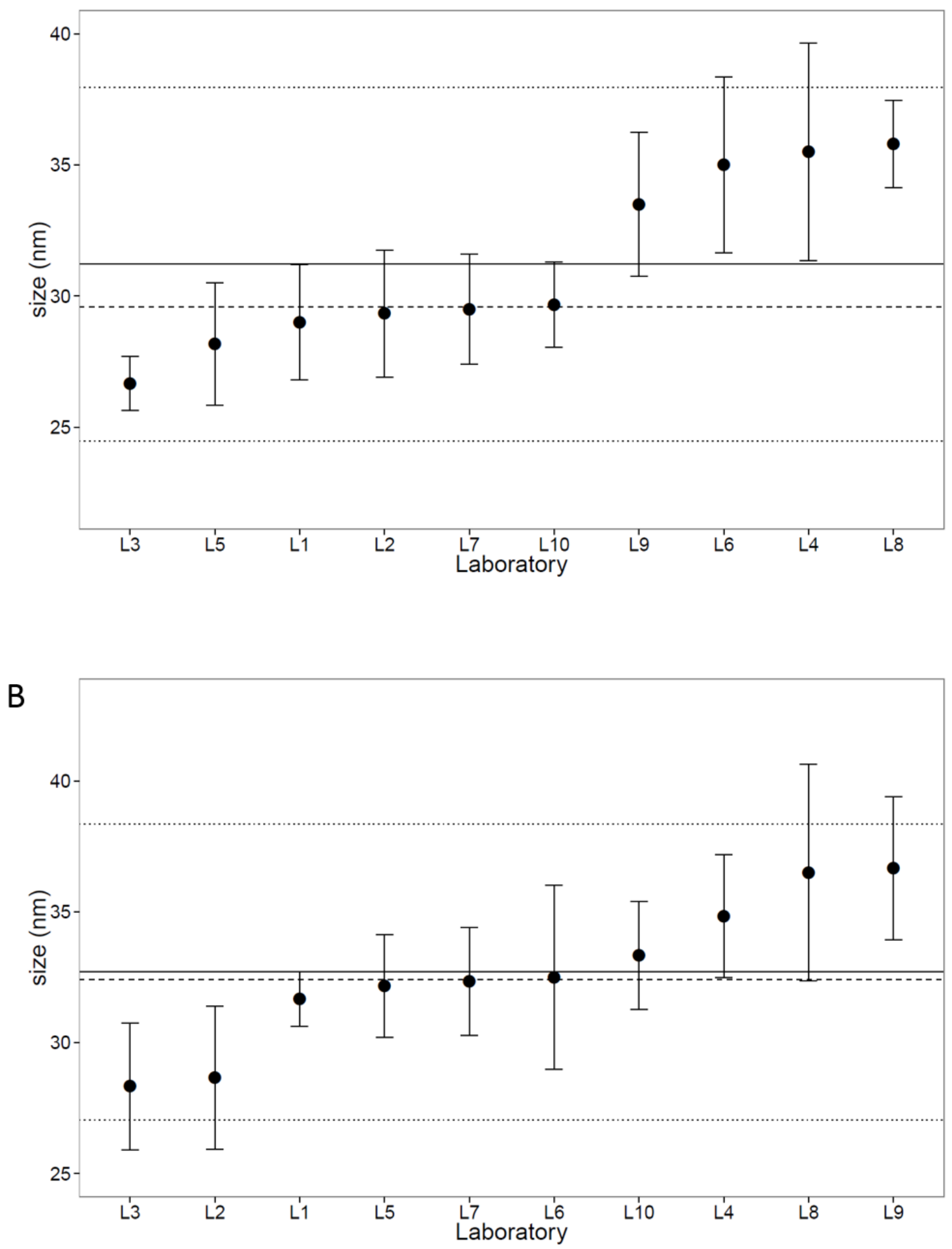

Figure 4 
A

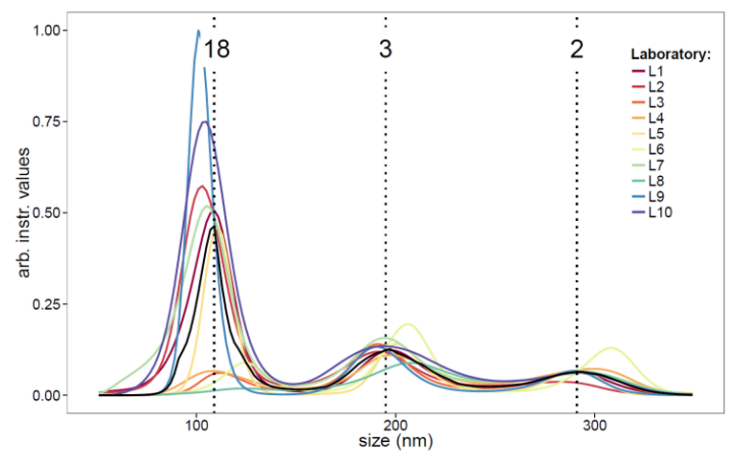

C

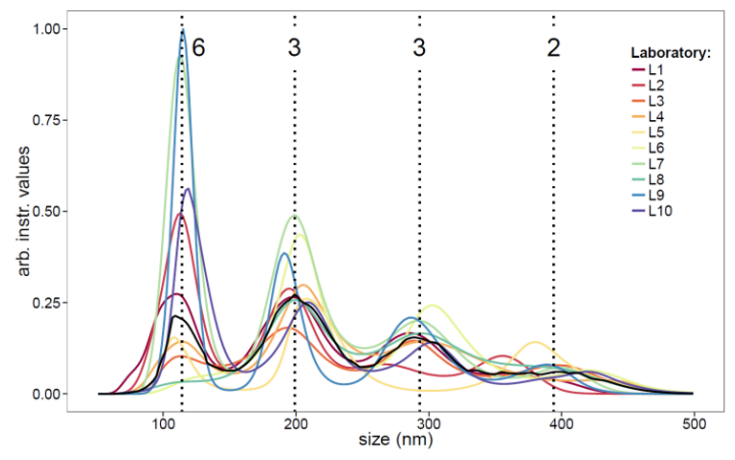

B

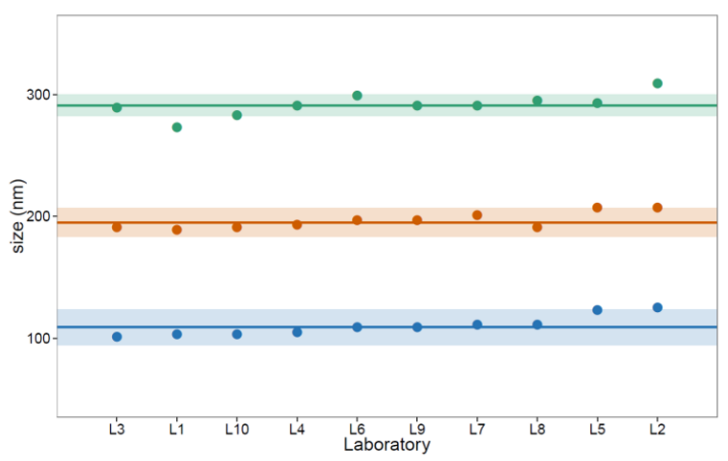

D

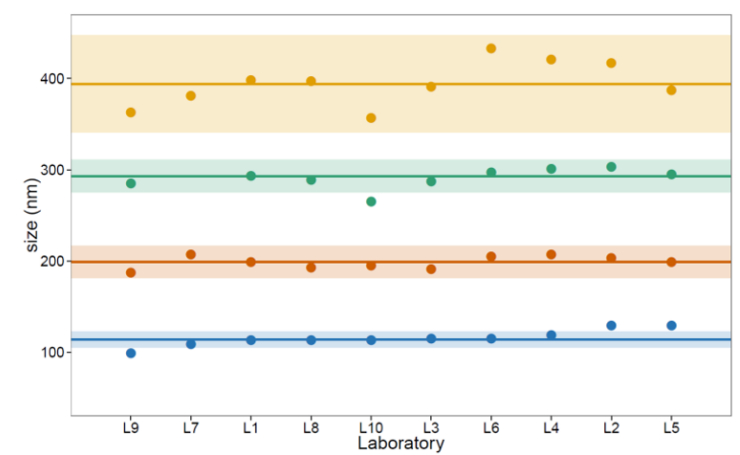

Figure 5 


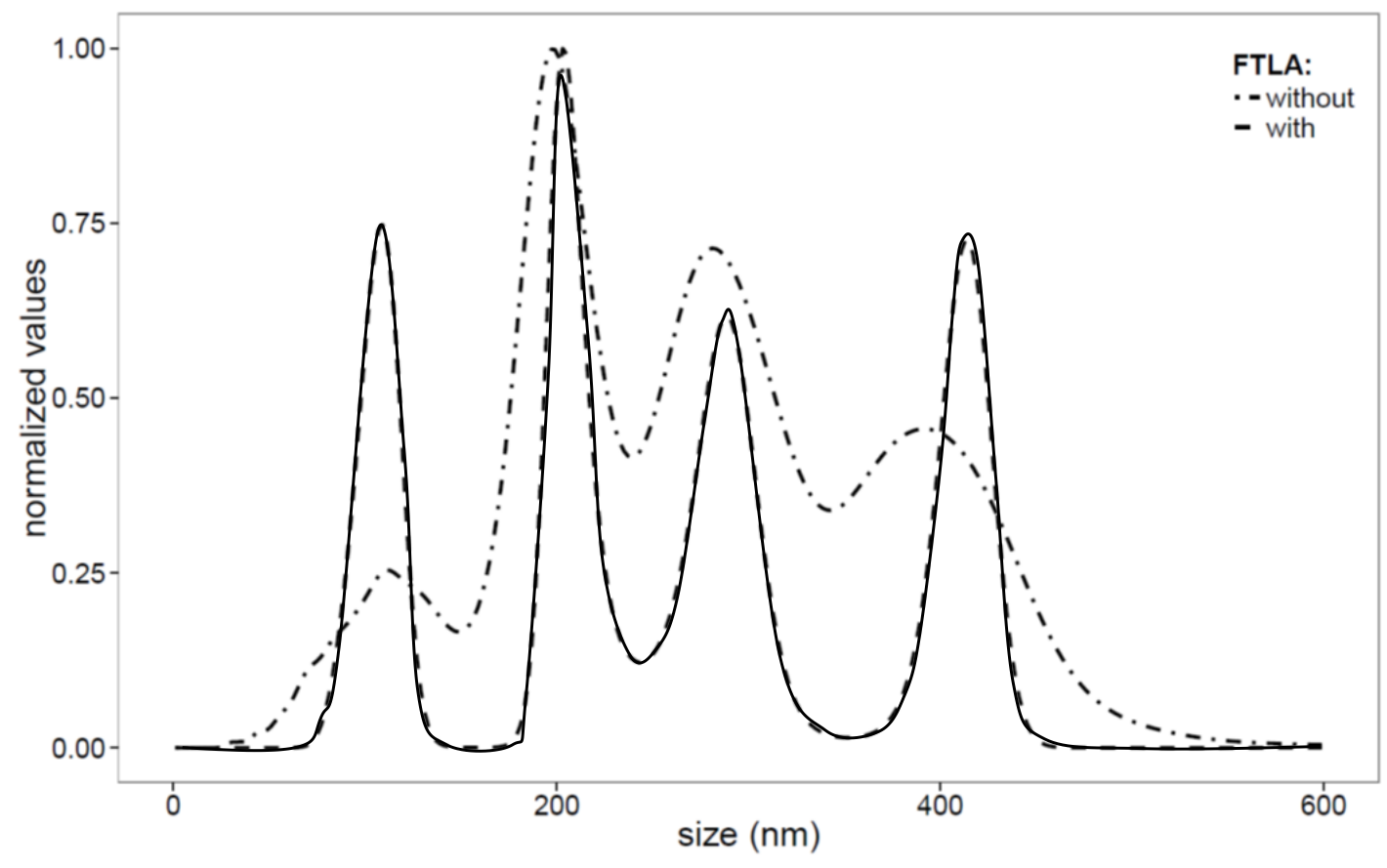

Figure 6 
A

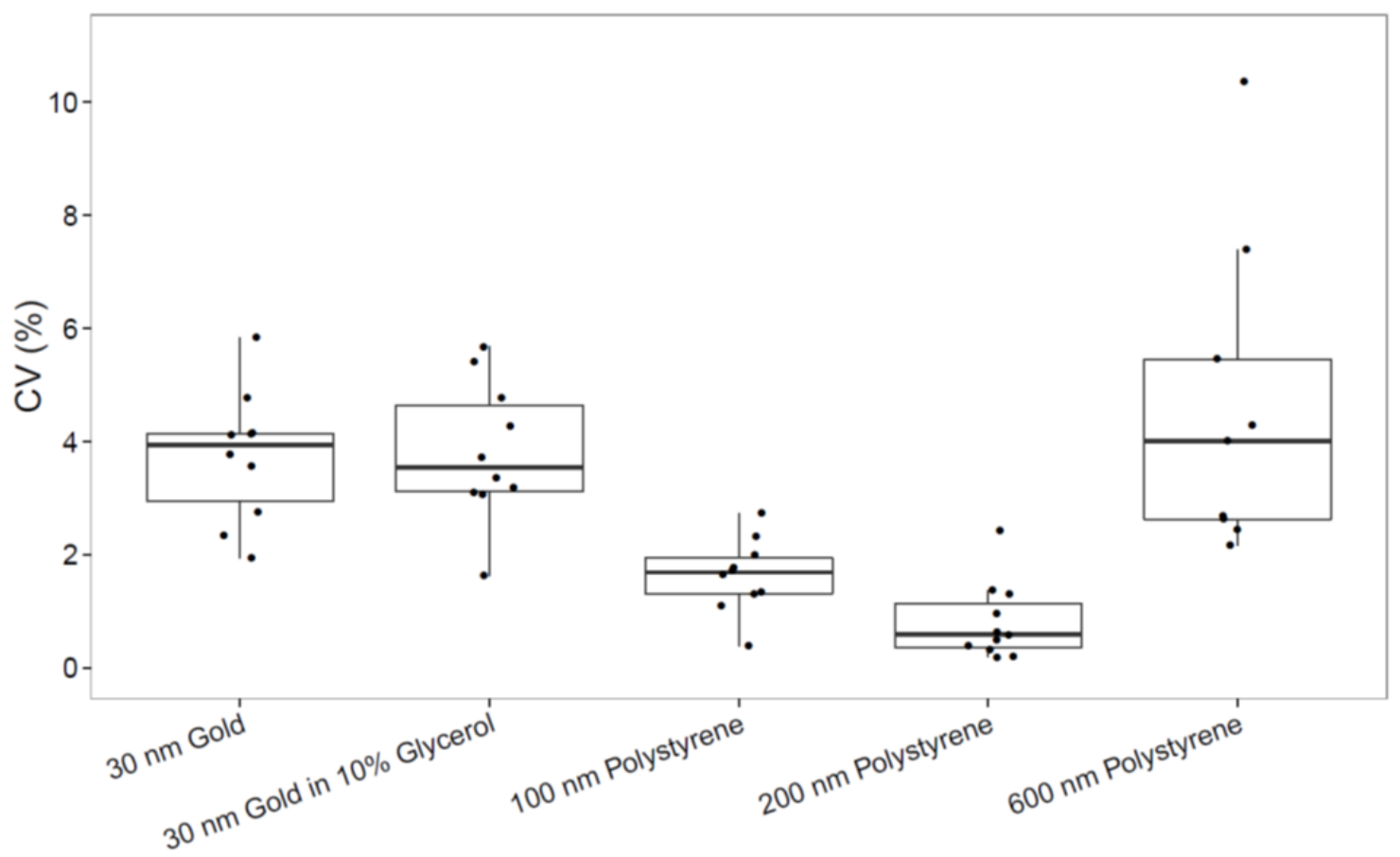

B

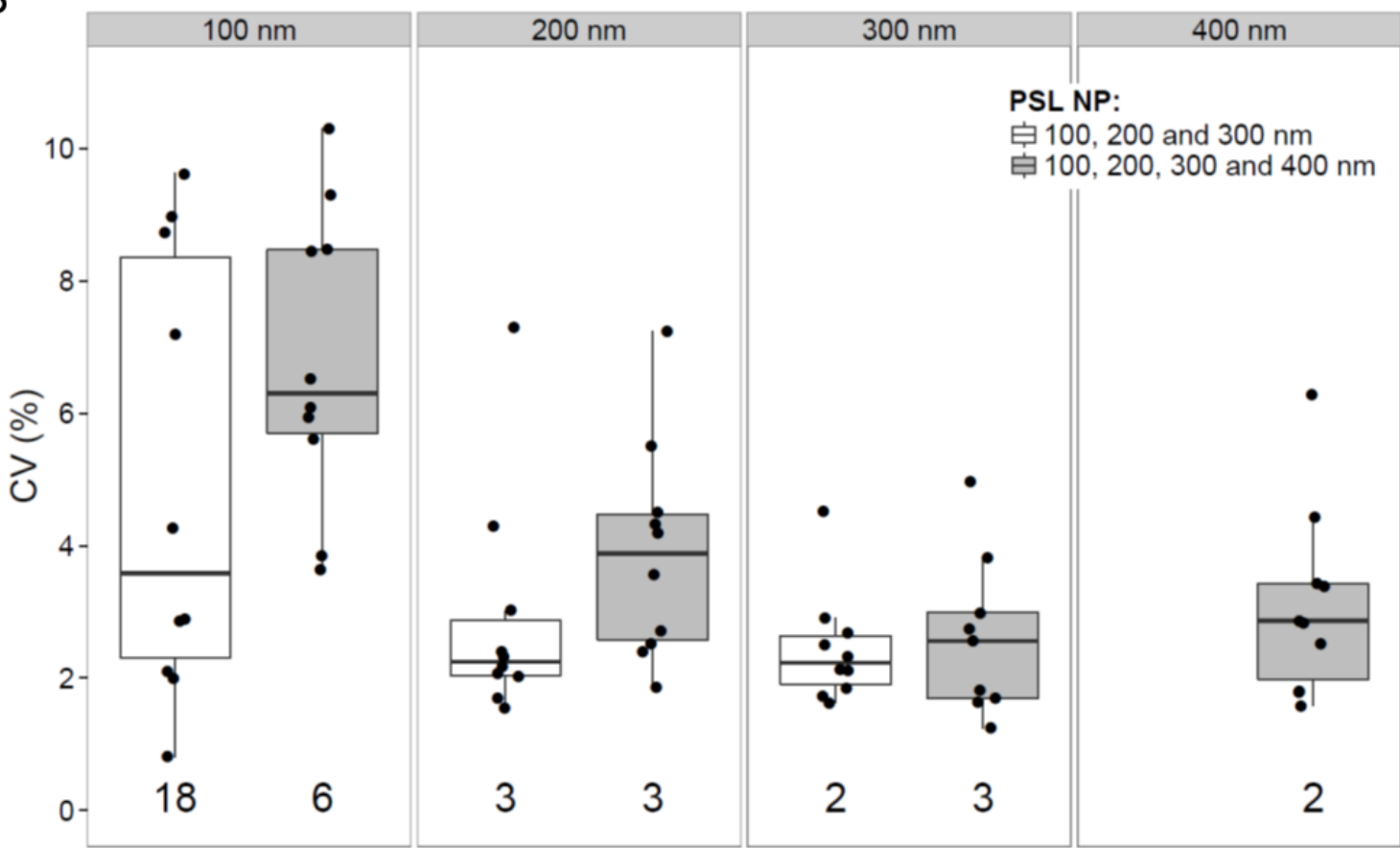

Figure 7 


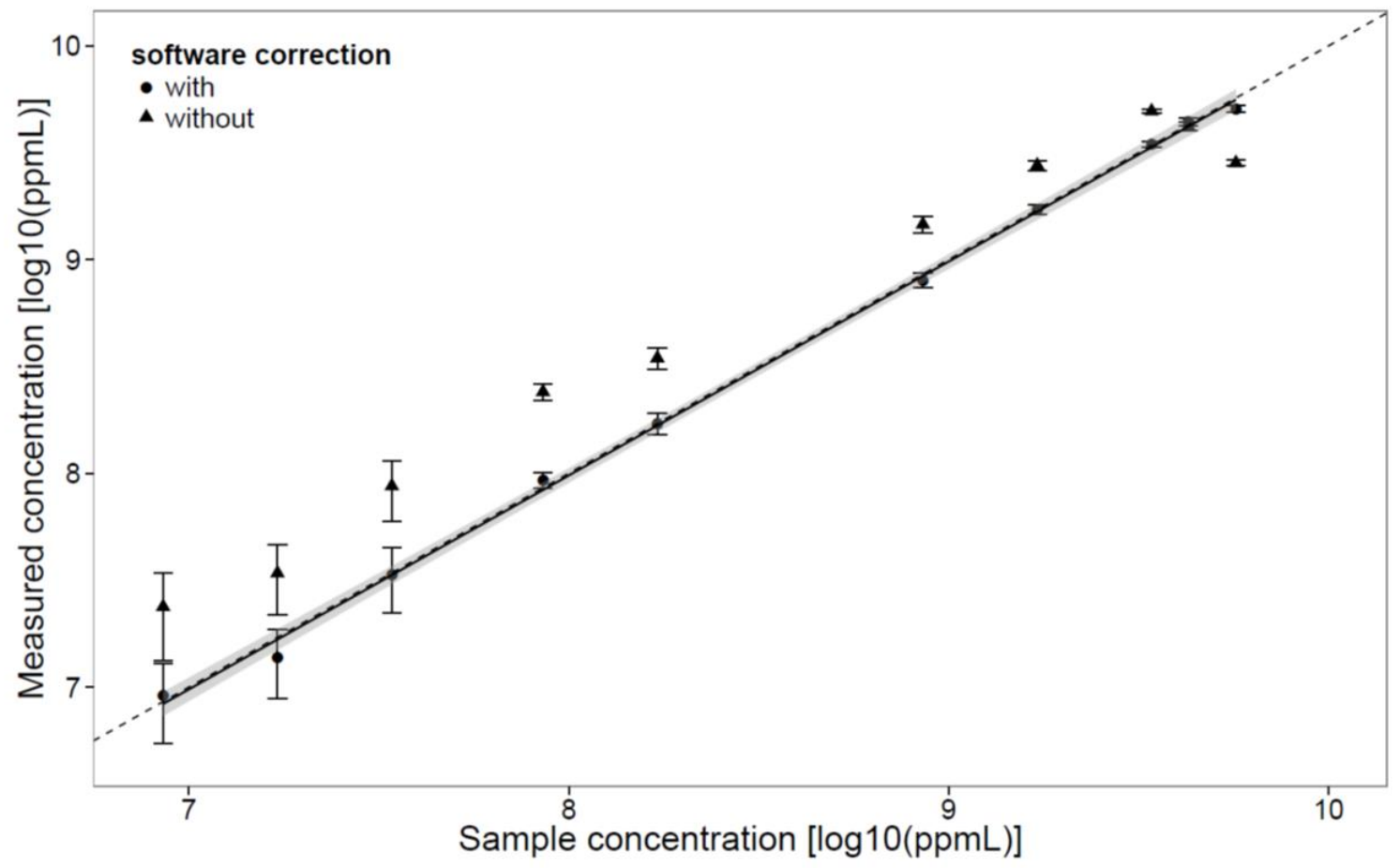

Figure 8 


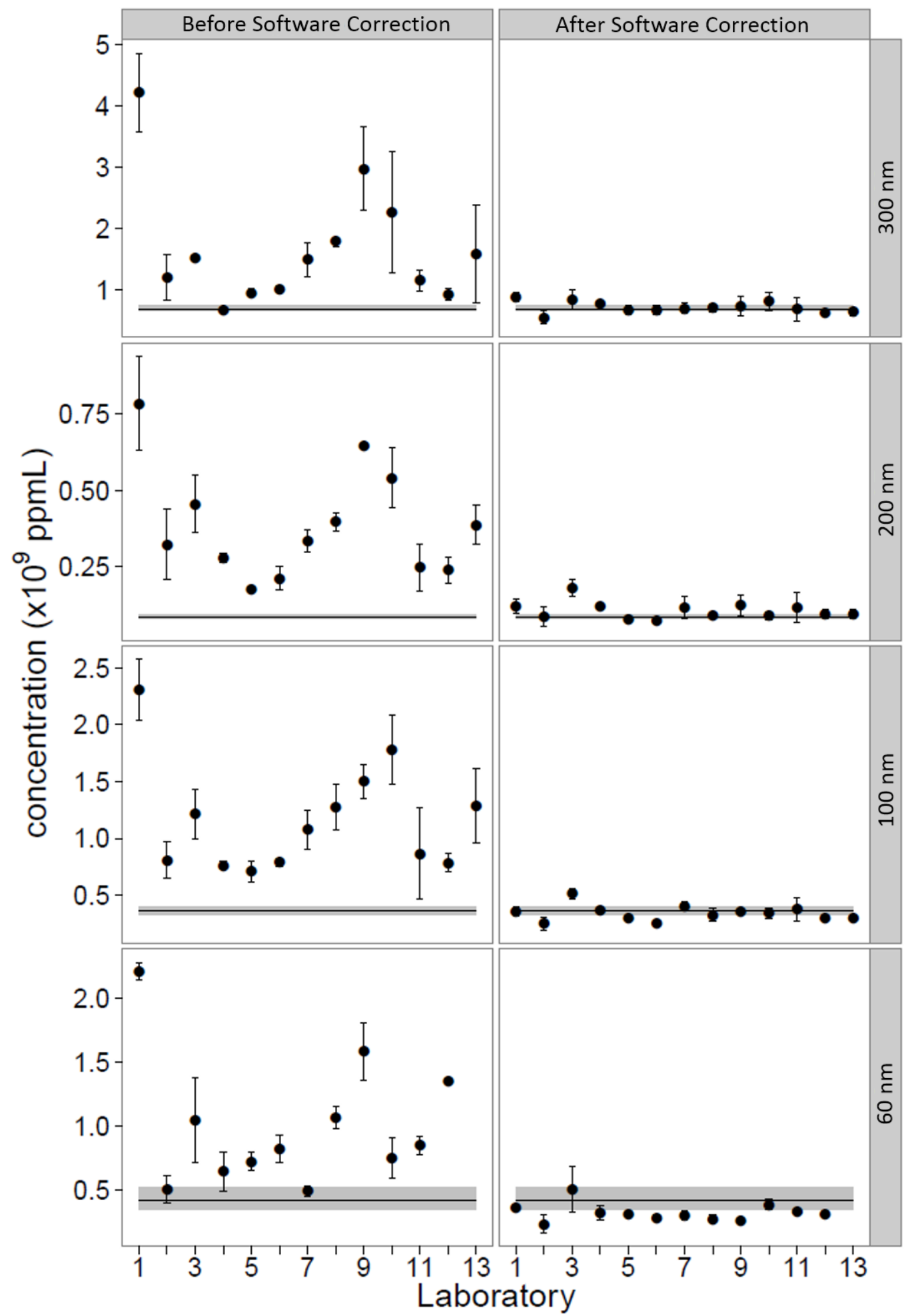

Figure 9 


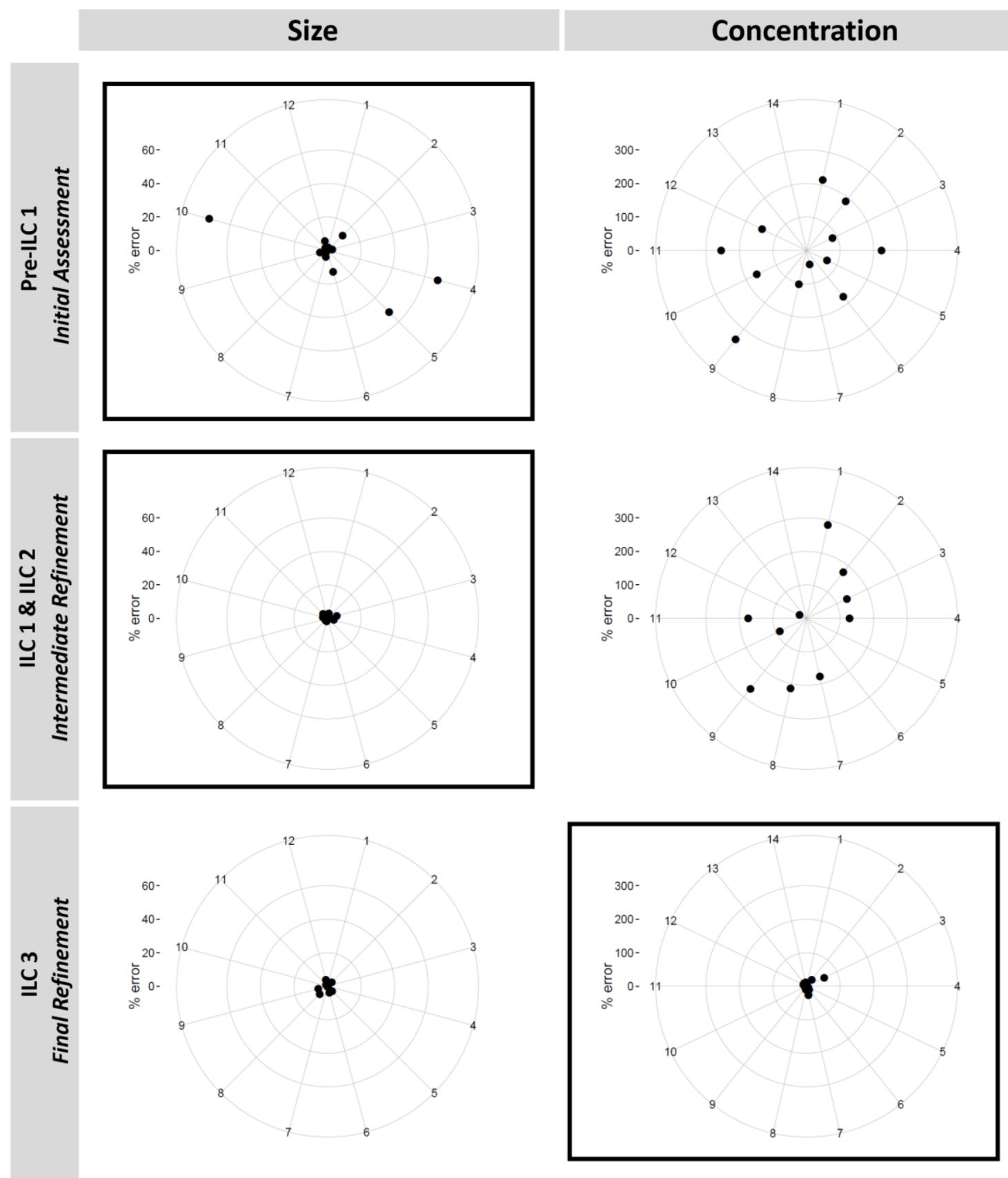

Figure 10 
Table 1

\begin{tabular}{lllcc}
\hline Participant & Platform & Camera & $\begin{array}{c}\text { Laser Wavelength } \\
(\mathbf{n m})\end{array}$ & $\begin{array}{c}\text { Temperature } \\
\text { Control }\end{array}$ \\
\hline $\mathbf{1}$ & LM10 & sCMOS & 532 & Yes \\
$\mathbf{2}$ & NS500 & EMCCD & 405 & Yes \\
$\mathbf{3}$ & NS500 & sCMOS & 405 & Yes \\
$\mathbf{4}$ & NS500 & sCMOS & 405 & Yes \\
$\mathbf{5}$ & LM20 & CCD & 635 & No \\
$\mathbf{6}$ & LM10 & CCD & 635 & No \\
$\mathbf{7}$ & LM10 & CCD & 635 & No \\
$\mathbf{8}$ & LM20 & CCD & 635 & No \\
$\mathbf{9}$ & NS500 & sCMOS & 405 & Yes \\
$\mathbf{1 0}$ & LM20 & CCD & 635 & No \\
$\mathbf{1 1}$ & LM10 & EMCCD & 405 & Yes \\
$\mathbf{1 2}$ & LM10 & sCMOS & 405 & No \\
\hline
\end{tabular}


Table 2

\begin{tabular}{|c|c|c|c|c|c|}
\hline $\begin{array}{l}\text { Nominal } \\
\text { Size }\end{array}$ & Material & TEM Size & TEM SD & $\begin{array}{l}\text { TEM \% } \\
\text { CV }\end{array}$ & DLS Size \\
\hline $100 \mathrm{~nm}$ & PSL & $\begin{array}{l}102 \mathrm{~nm}+/-3 \\
\mathrm{~nm}\end{array}$ & $5.2 \mathrm{~nm}$ & 5.1 & $95-106 \mathrm{~nm}$ \\
\hline $200 \mathrm{~nm}$ & PSL & $\begin{array}{l}203 \mathrm{~nm}+/-5 \\
\mathrm{~nm}\end{array}$ & $4.7 \mathrm{~nm}$ & 2.3 & $197-212 \mathrm{~nm}$ \\
\hline $300 \mathrm{~nm}$ & PSL & $\begin{array}{l}296 \mathrm{~nm}+/-6 \\
\mathrm{~nm}\end{array}$ & $5.3 \mathrm{~nm}$ & 1.8 & $296-310 \mathrm{~nm}$ \\
\hline $400 \mathrm{~nm}$ & PSL & $\begin{array}{l}400 \mathrm{~nm}+/-9 \\
\mathrm{~nm}\end{array}$ & $7.3 \mathrm{~nm}$ & 1.8 & $398-430 \mathrm{~nm}$ \\
\hline $600 \mathrm{~nm}$ & PSL & $\begin{array}{l}600 \mathrm{~nm}+/-9 \\
\mathrm{~nm}\end{array}$ & $10 \mathrm{~nm}$ & 1.7 & Not available \\
\hline $30 \mathrm{~nm}$ & $\mathrm{Au}$ & $30.6 \mathrm{~nm}$ & $\begin{array}{l}\text { Not } \\
\text { available }\end{array}$ & $<8 \%$ & Not available \\
\hline
\end{tabular}


Table 3

\begin{tabular}{lll}
\hline $\begin{array}{l}\text { Specified Particle } \\
\text { Size } \pm \text { error }\end{array}$ & $\begin{array}{l}\text { Mean Particle Size } \pm \text { Standard } \\
\text { Deviation }\end{array}$ & \% CV (mean size) \\
\hline $60 \pm 4 \mathrm{~nm}$ & $65.29 \pm 2.83 \mathrm{~nm}$ & 4.34 \\
$100 \pm 3 \mathrm{~nm}$ & $102.64 \pm 2.88 \mathrm{~nm}$ & 2.81 \\
$203 \pm 5 \mathrm{~nm}$ & $201.51 \pm 6.33 \mathrm{~nm}$ & 3.15 \\
$296 \pm 6 \mathrm{~nm}$ & $281.15 \pm 14.70 \mathrm{~nm}$ & 5.23 \\
\hline
\end{tabular}


Table 4

\begin{tabular}{lllll}
\hline Round & $\begin{array}{l}\text { Size }(\% \\
\text { error })\end{array}$ & $\begin{array}{l}\text { Size }(\% \\
\text { CV })\end{array}$ & $\begin{array}{l}\text { Concentration } \\
(\% \text { error })\end{array}$ & $\begin{array}{l}\text { Concentration } \\
(\% \mathbf{C V})\end{array}$ \\
\hline $\begin{array}{l}\text { Pre-ILC } \\
\text { ILC 1 and }\end{array}$ & 20 & 33.3 & 170 & 15.7 \\
ILC 2 & 2.2 & 3.2 & 170 & 11.6 \\
ILC 3 & 2.6 & 2.8 & 10.5 & 8.6 \\
\hline
\end{tabular}

